\title{
Improving the Central Santos Basin pre-salt understanding by reimaging multi-azimuth data
}

Marcela Ortin*, Raul Ysaccis, Hongyan Li, Pedro Barros, Schlumberger.

Copyright 2021, SBGf - Sociedade Brasileira de Geofísica

This paper was prepared for presentation during the $17^{\text {th }}$ International Congress of the Brazilian Geophysical Society held in Rio de Janeiro, Brazil, 16-19 August 2021.

Contents of this paper were reviewed by the Technical Committee of the $17^{\text {th }}$ International Congress of the Brazilian Geophysical Society and do not necessarily represent any position of the SBGf, its officers or members. Electronic reproduction or storage of any part of this paper for commercial purposes without the written consent of the Brazilian Geophysical Society is prohibited.

\section{Abstract}

The first oil discovery in the Brazil pre-salt was the giant Lula field in 2006. At that time, the only available seismic data in the area were 2D lines and narrow-azimuth, azimuth towed-streamer. Today with Santos Basin having one of the ten biggest oil reserves in the world, more accurately imaged seismic data are required to better understand the producing fields and to facilitate the development of others. In this work, we show how we can combine already acquired surveys to generate multiazimuth data to enable a better understanding of the Central Santos area.

\section{Introduction}

A complex geological setting, such as the variable thickness and stratified evaporites characteristic of the Santos Basin, observed in Figure 1, requires good-quality seismic data to better delineate the pre-salt target. It is desirable to have wide- or full-azimuth data to achieve a more uniform illumination, but those datasets are not commonly available offshore Brazil. To generate an improved image with existing data, up to four previously acquired surveys were combined in this project. This multi-azimuthal information helped generate a more representative multiple model, a more robust earth model, and a final image with better subsurface illumination.

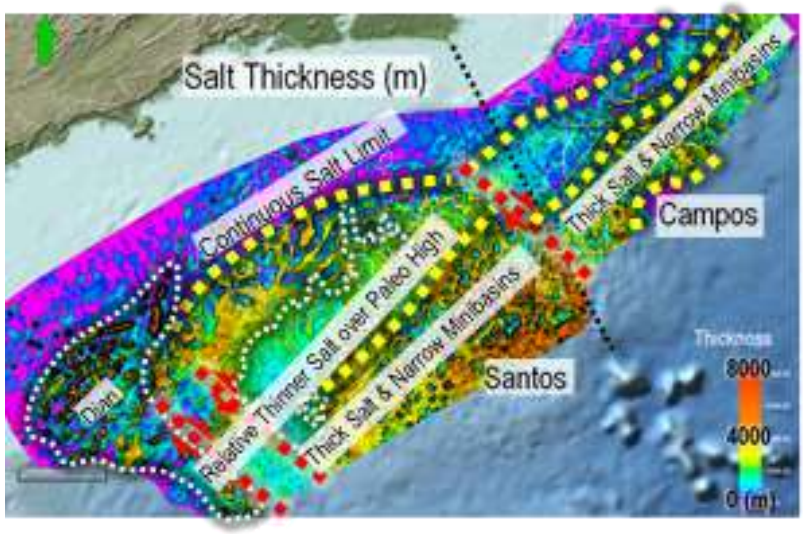

Figure 1 - Salt thickness map in the Santos and Campos Basins. The complexity and variation of the evaporites can be observed.
The improved illumination resulting from having different azimuths contributing is of great importance in areas with complex geological formations such as in the presence of evaporites. The information from narrow-azimuth (NAZ) surveys acquired with different azimuths travels along different paths in the overburden and sees a different image of the top of salt when this is complex. In the same way, the different wavefields cross the carbonate faults in different directions in the pre-salt. When all of this information is used simultaneously to update the velocity model, using either tomography or full-waveform inversion (FWI), this results in a more robust model than one generated by a single NAZ survey processed in isolation (Howard and Moldoneanu, 2006). Having a more accurate model directly translates to a more reliable image.

\section{Datasets}

The project combined three NAZ datasets and one fullazimuth survey, Figure 2. The primary dataset was acquired between 2012 and 2014 with a $123^{\circ}$ azimuth. The towed-streamer spread consisted of 12 streamers with an 8-km maximum offset and 50-m streamer separation. The signal processing and imaging finished in 2015. The second dataset utilized the same configuration but was acquired with an azimuth of $158^{\circ}$. The third dataset was a 2002 acquisition with $100 \mathrm{~m}$ between

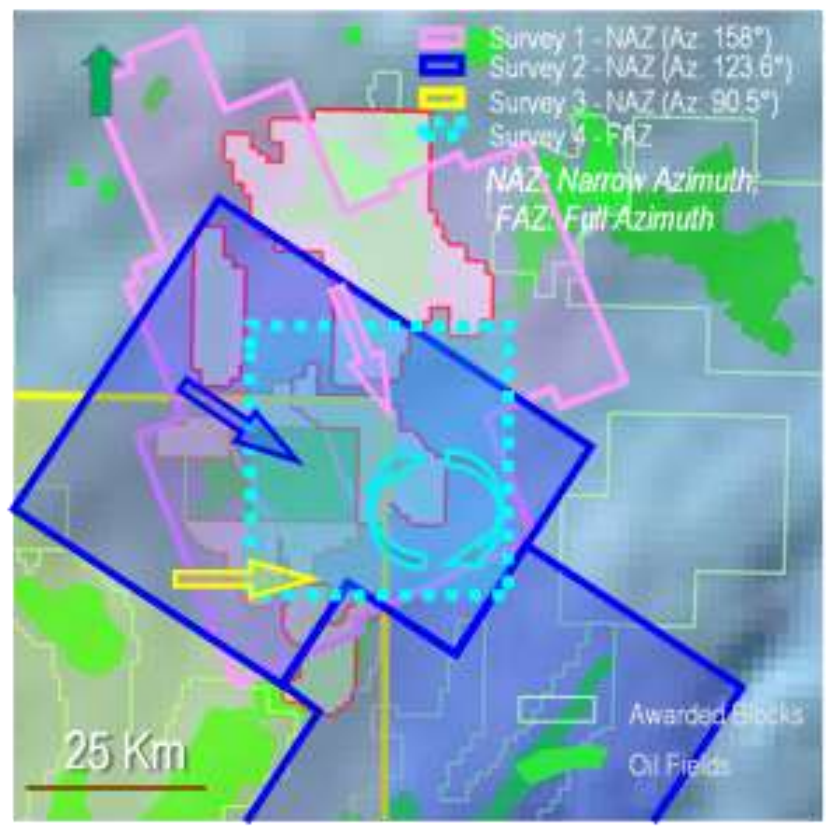

Figure 2 - Map showing the different surveys used in the project and their correspondent azimuths. 
streamer cables, $6 \mathrm{~km}$ maximum offset and shot in an east-west direction. Covering a section of this area is a full-azimuth survey with $8-\mathrm{km}$ maximum offset that was acquired in 2011 using a circular shooting geometry with the processing finished in 2012 (Cooke et al., 2012).

The NAZ surveys, although not being the product of a planned multi-azimuth survey design study, have sufficient azimuthal variation to complement each other and enhance the subsurface illumination.

\section{Signal processing}

Three of the datasets were severely affected by lowfrequency swell noise. Using contemporary noise attenuation techniques increased the signal-to-noise ratio significantly compared to the previous processing. This allowed the adaptive deghosting (Rickett, 2014) to recover the low frequencies that are extremely valuable to image the pre-salt structures and, particularly, to enhance the fault definition.

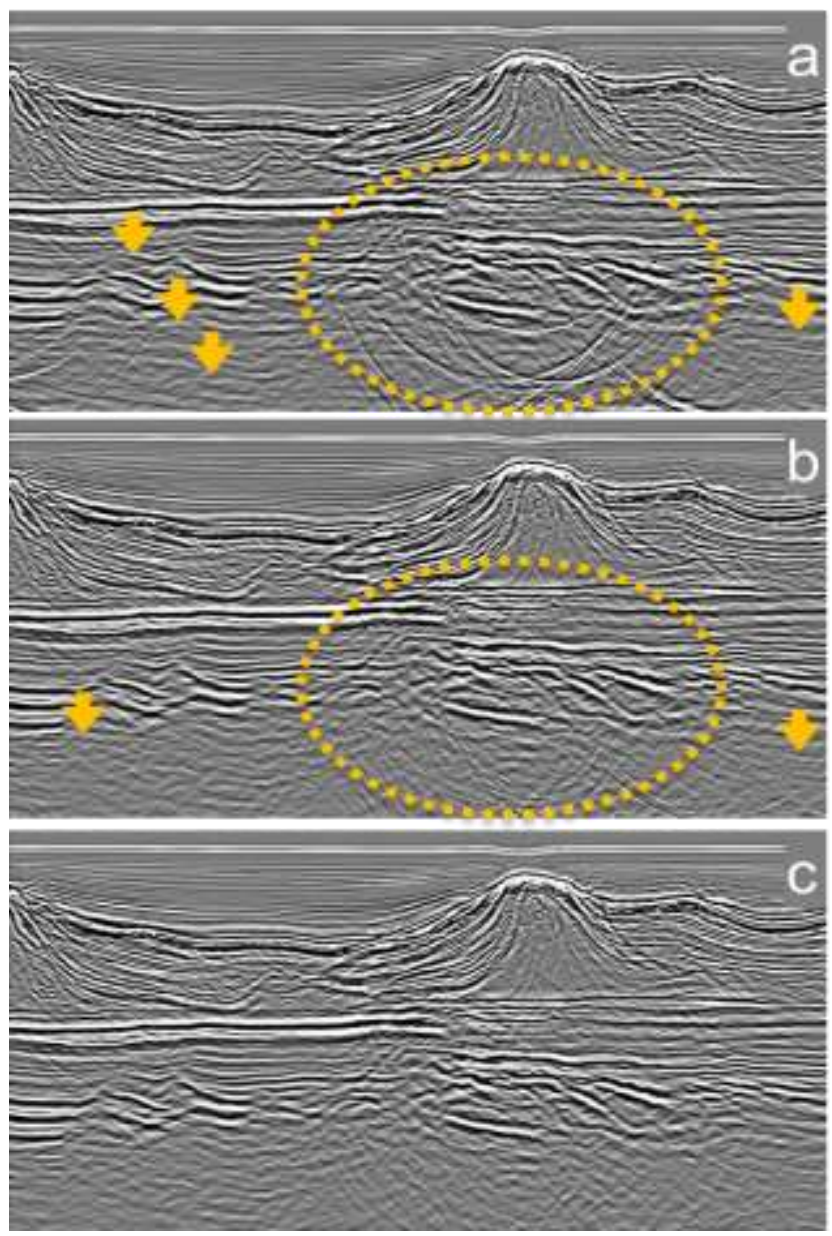

Figure $3-2 D$ reverse time migration images comparing a) data before demultiple application, b) data with constant aperture $3 D$ SRME applied, and c) data with variable aperture and multiazimuthal information for 3D SRME model estimation. Marked in yellow are the main multiples present in the different images. It is clear how the demultiple improves by using multi-azimuth data and variable aperture to attenuate, in particular, the complex topof-salt-related multiples marked in the dashed circle.
The free surface multiples are strong in the area, not only the ones related to the smooth water bottom, but also those generated in the complex top of salt. These latter ones are more challenging to model because of the generator geometry. Two main factors contributed to derive a robust model: having data with different azimuths and using a variable aperture 3D surface-related multiple elimination (3D SRME). This allowed using up to a 4.3-km aperture in inline and crossline directions when needed, but also maintaining the workflow efficiency (Espinoza et al., 2017). In Figure 3, one can observe the uplift produced by the combination of these two approaches.

In the Santos Basin, the interbed multiples are a wellknown challenge, with the stratified salt being the main generator. Combining data with different azimuths helps to attenuate them, based on their different expression in the different azimuths. The possibility of doing interbed modelling in the data domain and subtraction in the image domain is there if they are still considered to affect the interpretation of the target in the final image.

\section{Model building}

To start the model building, the legacy model was analyzed and preconditioned accordingly. The shortwavenumber details were removed, leaving a representative background trend. The anisotropy was validated, and a simpler trend introduced. To have a unique model that can explain the data from all the different surveys with their corresponding azimuths, a high-resolution, multi-azimuth common image point (CIP) tomography (Woodward et al., 2008) was used to update the post-salt section. With this the residual moveout from the Kirchhoff depth migration gathers in the different directions was minimized. The next step was a multisurvey least-squares full-waveform inversion to better define details in the overburden. Having the different azimuths allowed for a more-detailed model of the post-salt section. A final pass of the CIP tomography was run to guarantee gather flatness. The cumulative velocity variation in the post-salt in Figure 4 shows a nice correlation of the update with the geology. The velocity

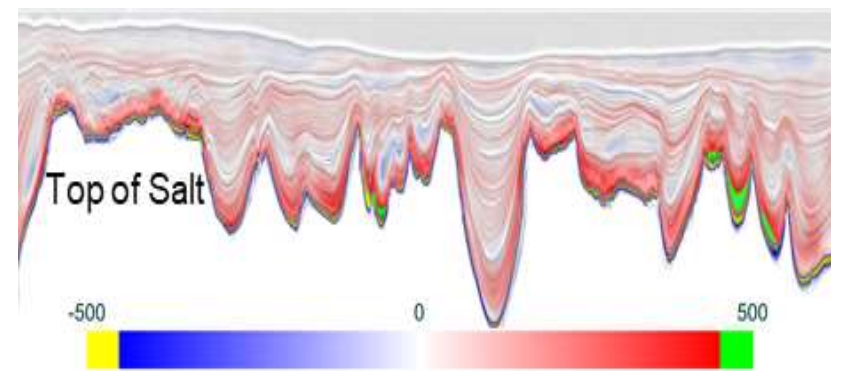

Figure 4 - Total velocity update in the post-salt section, product of multiple CIP tomography and FWI updates. Good correlation of the updates with the geology. Significant velocity increase above the top of salt, capturing the Albian carbonates high velocity.

change along the faults is well captured in the update, as well as the high velocities corresponding to the Albian carbonates. The post-salt model was validated with the well information and with residual moveout (RMO) maps in different windows. In Figure 5, the RMO map of the 
final post-salt model was estimated in a window from water bottom to the top of the Albian carbonates for three of the surveys and compared with the initial RMO of one of the surveys. The final RMO has small values and a very short wavelength, indicating that the gather flatness was achieved in the sediment window. Another thing to highlight is the comparable RMO behavior within the different surveys. The main goal when dealing with multiazimuth data is to derive a single model that explains the

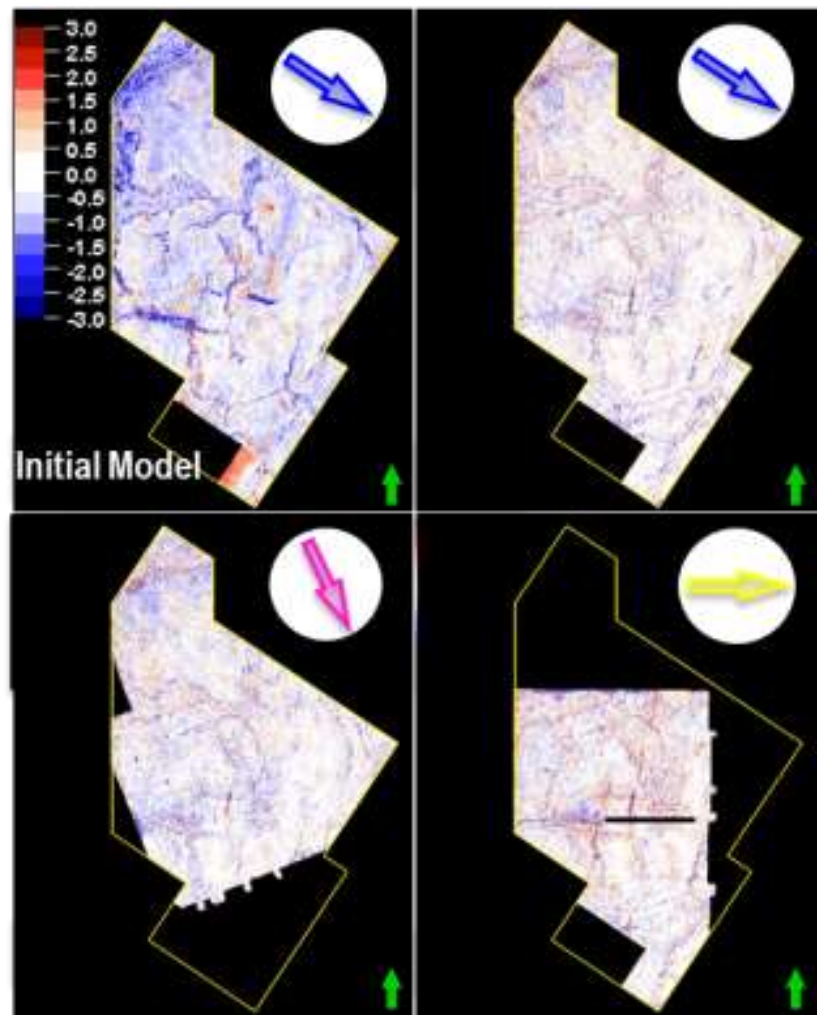

Figure 5 - RMO map calculated within the post-salt sediment window for three of the surveys independently. On the top left, the initial RMO map in the mentioned window for comparison. The RMO for the three surveys is small and of a very short wavelength, showing the good level of detail captured in the model.

data for all of the different azimuths. After all these validations, the top-of-salt interpretation started. There are no major overhangs in the area, but a detailed interpretation in the zones where the top of evaporites is more complex did improve the continuity of the base of salt compared with the legacy. The initial salt velocity was generated using a smooth trend that honors the well information and the seismic image coherency used to delineate the areas with halite and the ones with stratified anhydrite. For the pre-salt, a trend based in the regional geological knowledge was introduced, considering the high-velocities carbonates and the slower clastic section underneath. A smooth transition between the two zones was included to update the two intervals simultaneously. To solve for the variation in velocities in the evaporite interval and to add detail in the pre-salt section, reflection full-waveform inversion followed by multi-azimuth CIP tomography using reverse time migration (RTM) subsurface angle azimuth gathers were used (Vyas et al., 2011).

Borehole information was used throughout the model building. Starting with the anisotropy estimation and presalt velocity trend definition, followed by misties analysis through markers and interpretation and surface and synthetics seismic comparison after each model update.

\section{Results}

To generate the final image, each survey was migrated separately using both Kirchhoff and RTM algorithms and combined afterwards.

The value of the multi-azimuth and the reprocessing is clear in Figure 6, where the 45-Hz RTM image of each of the independent surveys is compared with the combination of them and with the legacy Kirchhoff image. To facilitate the comparison, traffic-light color-coded arrows were included. From the analysis of the pictures some conclusions can be drawn.

Firstly, the data character is very similar, despite their differing acquisitions, owing to carefully tailored processing sequences adjusted for each survey requirement. Secondly, each independent image shows areas of good imaging and poorer imaging related to the structure orientation and the preferential azimuth required to properly illuminate it. Thirdly the combined image is superior to the legacy everywhere, thanks to the new signal processing, a robust earth model, and a moresuitable algorithm to image the pre-salt.

\section{Conclusions}

Understanding the Santos Basin requires the best available data. Combining already acquired surveys with improvements in signal processing, earth model building, and imaging techniques generates a final image that is much improved over the legacy image in terms of resolution, signal-to-noise ratio, and amplitude consistency, which enables a better understanding of the pre-salt interval. In terms of signal processing, it is valuable to highlight the benefits of adaptive deghosting to generate a broadband dataset that helps in the pre-salt horizons continuity, fault definition, and the variable aperture and multi-azimuth 3D SRME to generate a robust free surface multiple model. In terms of model building, the main contributor was the multi-azimuthal data that were used simultaneously in the CIP tomography, least squares, and reflection FWI steps, allowing us to build a detailed earth model that honors the different datasets. Finalizing a 45-Hz RTM image brought all these improvements together to capture the pre-salt carbonate details.

\section{Acknowledgments}

The authors thank WesternGeco MultiClient for permission to present this work as well as the members of the project team. 


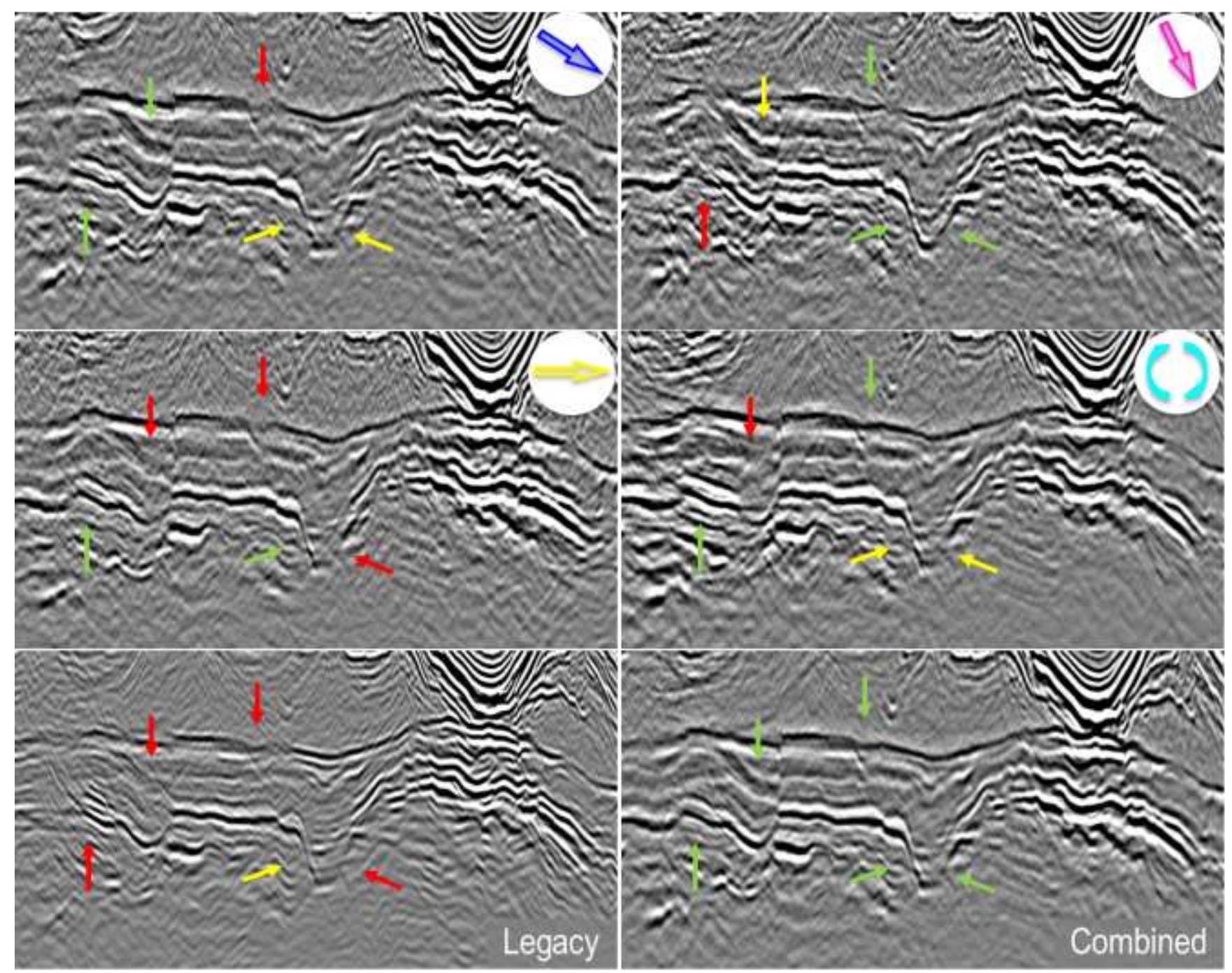

Figure 6 - Comparison of the final 45-Hz RTM images for the four surveys independently (top four) and the legacy and final combined image on the bottom. The traffic-light color-coded arrows signal where the image has the best quality between the different products (green), where the image has enough definition (yellow), and where the image is below the quality of the other images (red).

None of the surveys alone has a superior image everywhere as expected due the variation of the structures and top-of-salt orientation. The combined multi-azimuth image has a superior image than the legacy in the whole pre-salt section.

\section{References}

COOKE, A., Le DIAGON, F., De MARCO, R., AMAZONAS, D., BUNTING, T., MOLDOVEANU, N., and MATTOS, E. 2012. Full-azimuth towed-streamer seismic: An exploration tool for pre-salt hydrocarbon exploration offshore Brazil. 82nd Annual International Meeting, SEG, Expanded Abstracts, 1-5.

ESPINOZA, C., SANGER, W., NICHOLS, D., and GRANIEL, J. 2017. Geology-constrained data-driven multiple prediction. 87th Annual International Meeting, SEG, Expanded Abstracts, 4747-4751.

HOWARD, M.S. and MOLDOVAEANU, N. 2006. Marine survey design for rich-azimuth seismic using surface streamers. 76th Annual International Meeting, SEG, Expanded Abstracts, 2915-2919.
RICKETT, J. 2014. Successes and challenges in 3D interpolation and deghosting of single-component marine streamer data. 84th Annual International Meeting, SEG, Expanded Abstracts, 3599-3604.

VYAS, M., NICHOLS, D., and MOBLEY, E. 2011. Efficient RTM angle gathers using source directions. 81st Annual International Meeting, SEG, Expanded Abstracts, 31043108.

WOODWARD, M., NICHOLS, D., ZDRAVEVA, O., WHITFIELD, P. and Johns, T. 2008. A decade of tomography. Geophysics, 73, 5-11. 